\title{
Status quo palliativmedizinischer Tageskliniken und Tages- hospize in Deutschland: Ergebnisse einer gemischt-methodischen Studie
}

\section{Status quo of Palliative Day-Care Clinics and Day Hospices in Germany: Results of a Mixed-Methods Study}

\section{다(1) $(\Theta$}

\author{
Autoren \\ Beate Apolinarski ${ }^{*}$, Franziska A. Herbst ${ }^{*}$, Hanna A. A. Röwer, Nils Schneider, Stephanie Stiel
}

Institut

Institut für Allgemeinmedizin, Medizinische Hochschule Hannover, Hannover, Deutschland

Schlüsselwörter

Bestandsanalyse, Palliativversorgung, Hospizversorgung, teilstationäre Versorgung, Versorgung am Lebensende

Keywords

availability of health services, palliative care, hospice care, day care, terminal care

Bibliografie

Z Palliativmed 2021; 22: 215-224

DOI 10.1055/a-1514-8871

ISSN 1615-2921

(c) 2021. The Author(s).

This is an open access article published by Thieme under the terms of the Creative Commons Attribution-NonDerivative-NonCommercial License, permitting copying and reproduction so long as the original work is given appropriate credit. Contents may not be used for commercial purposes, or adapted, remixed, transformed or built upon. (https://creativecommons.org/licenses/by-nc-nd/4.0/)

Georg Thieme Verlag KG, Rüdigerstraße 14,

70469 Stuttgart, Germany

Korrespondenzadresse

Dr. Franziska A. Herbst, Institut für Allgemeinmedizin,

Medizinische Hochschule Hannover,

Carl-Neuberg-Straße 1, 30625 Hannover, Deutschland herbst.franziska@mh-hannover.de

\section{ZUSAMMENFASSUNG}

Ziel der Studie Der Bestand von Tageshospizen und palliativmedizinischen Tageskliniken in Deutschland soll erstmals systematisch erhoben und analysiert werden.

Methodik In dieser gemischt-methodischen Studie wurden Einrichtungsleitungen in leitfadengestützten Interviews
(06.-07.2020) und standardisierter Fragebogenerhebung (08.-10.2020) zu einrichtungsbezogenen Kriterien befragt. Interviewdaten wurden qualitativ inhaltsanalytisch, quantitative Daten deskriptiv ausgewertet.

Ergebnisse Die Interviews $(n=7)$ liefern einen Überblick über Organisation und Versorgungsangebot hospizlich-palliativer Tageseinrichtungen. Von 28 standardisiert befragten Einrichtungen waren 13 bereits in Betrieb. Die zumeist an andere Versorger angebundenen Einrichtungen bieten in der Regel zwischen 2 und 8 Tagesplätze an.

Schlussfolgerung Aufgrund der gezeigten Entstehungsdynamik scheint eine systematische Integration der neuen Strukturen in die bestehende Hospiz- und Palliativversorgung sinnvoll.

\section{ABSTRACT}

Objective The status of day hospices and palliative daycare clinics in Germany is systematically surveyed and analysed.

Methods In this mixed-method study, facility managers of hospice and palliative day-care facilities were questioned on facility-related criteria in guided interviews (06-07/2020) and via a questionnaire survey (08-10/2020). Interview data were analysed using the methodological principles of qualitative content analysis; quantitative data were analysed using descriptive statistics.

Results The interviews $(n=7)$ provide an overview of organisation and care services of hospice and palliative day-care facilities. Thirteen of the 28 facilities participating in the quantitative survey were already operating. The facilities are mostly affiliated with other service providers and usually offer between 2 and 8 day places.

Conclusion The authors recommend a systematic integration of these new, dynamic structures into existing hospice and palliative care.

* beide Autorinnen trugen in gleichem Maß zu dieser Arbeit bei 


\section{Einleitung}

Teilstationäre Einrichtungen zur Versorgung von Menschen mit nicht heilbaren Erkrankungen und begrenzter Lebenszeit bilden in Großbritannien, Irland und den USA neben den vollstationären und ambulanten Angeboten seit vielen Jahrzehnten einen wichtigen Versorgungspfeiler [1-5]. Bereits im Jahr 1975 nahm in Sheffield das erste Hospiz die teilstationäre Tagesversorgung auf [6]. Mitte der 1990er-Jahre verzeichnete Großbritannien über 200 teilstationäre hospizlich-palliative Angebote [1]. Primäre Ziele dieser Einrichtungen sind die Förderung der Selbstständigkeit und Verbesserung der Lebensqualität betroffener Patientinnen und Patienten in der Häuslichkeit, die Bewahrung vor sozialer Isolation sowie die Entlastung pflegender Angehöriger [3, 7, 8]. In anderen Ländern wie Frankreich oder Belgien hingegen, bestehen hospizlich-palliative teilstationäre Angebote erst seit den späten 1990er beziehungsweise 2000er Jahren $[9,10]$. Auch in Deutschland steckt die teilstationäre Hospiz- und Palliativversorgung noch in den Kinderschuhen [11, 12]. Hier kommen auf ca. 230 vollstationäre Erwachsenen-Hospize und ca. 330 Krankenhäuser mit Palliativstationen [13], wie der vorliegende Beitrag zeigen wird, gerade einmal 12 bestehende Tageshospize und sogar nur 5 palliativmedizinische Tageskliniken.

Diese Angebote sowie weitere, aktuelle Initiativen zum Aufbau von Tageshospizen und palliativmedizinischen Tageskliniken haben sich an einzelnen Standorten in Deutschland und aus der Praxis heraus gebildet. Der Aufbau und die Entwicklung dieser Einrichtungen erfolgten bislang jedoch unsystematisch. Zudem ist wenig darüber bekannt, welche Angebote die jeweiligen Einrichtungen abdecken, wie sie sich finanzieren und wie sie regional verteilt, strukturell aufgebaut und in hospizlich-palliative Netzwerke eingebunden sind. Da die individuellen Tagesangebote nicht zwangsläufig über Krankenkassen abgerechnet werden, besteht keine einheitliche Datenbasis, die dieses teilstationäre Versorgungsangebot in Deutschland hinreichend dokumentiert.

\section{Studienziel}

Mit der vorliegenden Studie soll die Frage nach dem Bestand und den Charakteristika palliativmedizinischer Tageskliniken und Tageshospize in Deutschland beantwortet werden. Die Studie ist Teil des Forschungsprojekts ABPATITE [14], dessen Ziel es ist, den Auf- und Ausbau von hospizlich-palliativen Tagesangeboten evidenzbasiert zu begleiten und die übergeordnete Steuerung zu fördern.

\section{Methodik}

\section{Studiendesign, Rekrutierung und Studienpopulation}

In der vorliegenden gemischt-methodischen Studie wurde ein sequenzielles exploratives Design gewählt [15-17].

Systematische Recherche: Vorbereitend wurde zur Identifikation bestehender und im Aufbau befindlicher palliativmedizinischer Tageskliniken und Tageshospize in Deutschland eine systematische Recherche anhand nationaler Suchportale $[18,19]$, freier Informationen im Internet sowie Anfragen bei Fachgesellschaften durchgeführt. Eingeschlossen wurden gemäß der „S3-Leitlinie Palliativmedizin für Patienten mit einer nicht heilbaren Krebserkrankung “ teilstationäre Einrichtungen, sofern sie „spezialisierte Angebote für ambulante Patienten mit einer nicht heilbaren Erkrankung und begrenzter Lebenszeit“ darstellten [11].

Qualitative Interviews: Im nächsten Teilschritt führten 3 Forscherinnen (BA, FH, HR) leitfadengestützte, halbstrukturierte Interviews [20] mit Einrichtungsleitungen der recherchierten palliativmedizinischen Tageskliniken und Tageshospize zur Erhebung relevanter einrichtungsbezogener Kriterien (06.07.2020) bis eine Datensättigung erreicht und ein Überblick über Charakteristika der Einrichtungen gewonnen wurde. Das Erreichen der Datensättigung wurde definiert als der Zeitpunkt in der Datenerhebung und -analyse, an dem neue Informationen aus den Interviews nur noch zu geringfügigen bzw. keinen Veränderungen der Codestruktur führten [21]. Zur Feststellung der Datensättigung wurden die Interviewtranskripte fortlaufend analysiert. Für die Interviews wurden Leitungen möglichst verschiedener Einrichtungen hinsichtlich Art der Einrichtung, Einrichtungsgröße, Bestehensdauer, städtischer/ländlicher Raum, Stadtgröße und Finanzierung ausgewählt. Alle Teilnehmenden wurden telefonisch nach schriftlicher Ankündigung zur Studienteilnahme eingeladen. Vor dem Interview wurden alle Teilnehmenden über Ziel und Zweck der Erhebung informiert und unterzeichneten eine datenschutzrechtliche Einwilligungserklärung mit Einverständnis zur Audioaufnahme. Bei der Rekrutierung kam es durch COVID-19-bedingte Personalbelastungen bei 2 Anfragen zu Absagen.

Quantitative Befragung: Um die Charakteristika palliativmedizinischer Tageskliniken und Tageshospize weiter zu differenzieren, wurden in einer quantitativen Befragung Einrichtungs- und Ausstattungsmerkmale erhoben. Die Ergebnisse der Interviews flossen in die Entwicklung des standardisierten Erhebungsbogens ein. Leitungspersonen der ermittelten palliativmedizinischen Tageskliniken und Tageshospize wurden nach telefonischer Aufklärung über das Studienziel um Teilnahme an der schriftlichen Befragung gebeten (08.-10.2020). Anschließend wurde ihnen der schriftliche Fragebogen sowie eine Woche später eine Erinnerungs-E-Mail zugesandt.

\section{Datenanalyse}

Alle Interviews wurden audioaufgezeichnet, verschriftlicht und mithilfe der Software MaxQDA nach den methodischen Prinzipien der qualitativen Inhaltsanalyse nach Mayring [22, 23] ausgewertet. Dabei wurden die Themen des Interviewleitfadens als A-priori-Kategoriensystem angenommen und sukzessive induktiv um Codes, Konzepte und Kategorien aus dem Interviewmaterial erweitert. Zwei Forscherinnen (BA, HR) codierten unabhängig voneinander die Interviews und konsentierten diese anschließend. Eine dritte Forscherin (FH) prüfte die Konsistenz der Codierungen.

Die quantitativ erhobenen Daten wurden in IBM SPSS Statistics 26 (SPSS Inc., Chicago, IL, USA) für Windows deskriptiv 


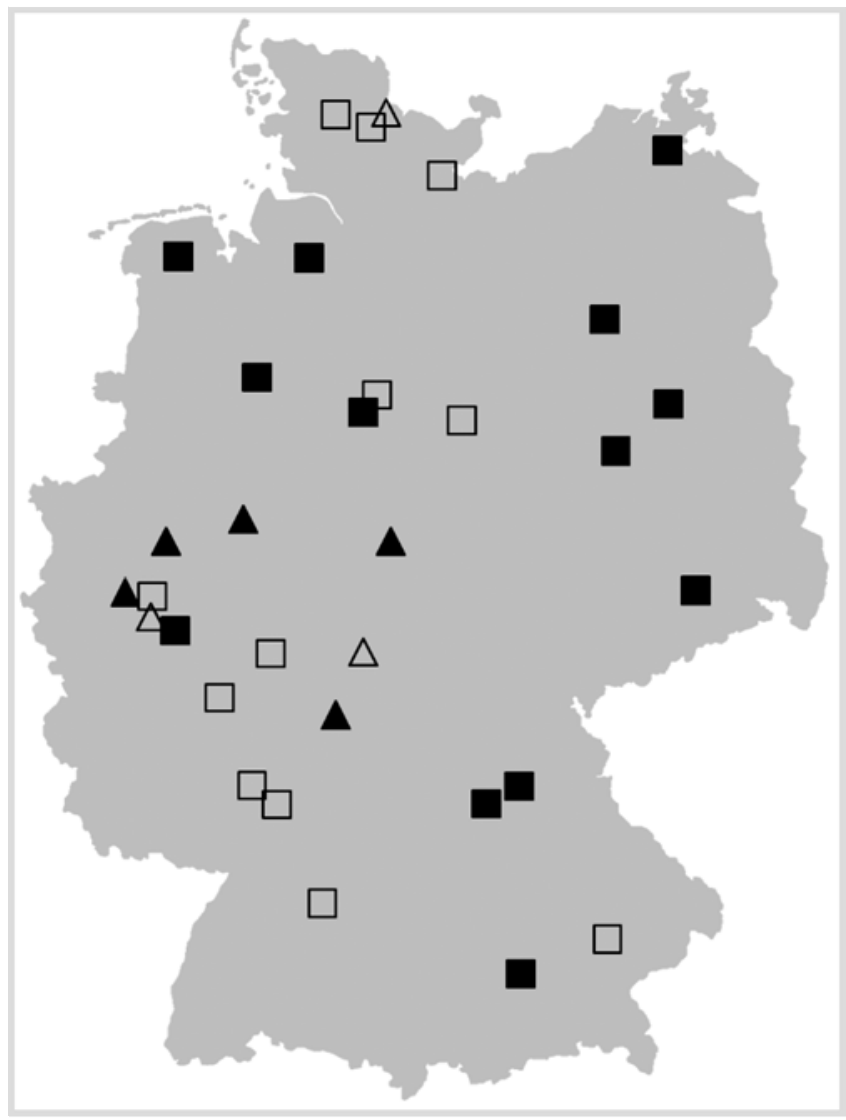

- Abb. 1 Verteilung geplanter und bestehender teilstationärer Einrichtungen in Deutschland. - Tageshospiz in Betrieb; $\square$ Tageshospiz in Planung; $\Delta$ Palliativmedizinische Tagesklinik in Betrieb; $\triangle$ Palliativmedizinische Tagesklinik in Planung.

nach Antworthäufigkeiten ausgewertet. Alle erhobenen Daten wurden anonymisiert gespeichert und verarbeitet.

\section{Ergebnisse}

Über die vorbereitende systematische Recherche konnten 25 Tageshospize und 8 palliativmedizinische Tageskliniken in Deutschland identifiziert werden ( $\triangleright$ Abb. 1 zur räumlichen Verteilung). Sie sind vorrangig in Großstädten und städtischen Kreisen zu finden $(n=25)$, seltener in ländlichen Regionen $(n=$ 8). Rund die Hälfte der Einrichtungen befindet sich zum Erhebungszeitpunkt im Aufbau oder in Planung (13 Tageshospize; 3 palliativmedizinische Tageskliniken).

\section{Ergebnisse der Codeanalyse der qualitativen Interviews mit Einrichtungsleitungen}

Gemäß des sequenziellen Vorgehens der Studie werden qualitative und quantitative Ergebnisse im Folgenden bewusst getrennt voneinander, nacheinander berichtet. An den Interviews nahmen 7 Einrichtungsleitungen teil (aus Tageshospizen: 4; aus palliativmedizinischen Tageskliniken: 3); davon wurden 6 Interviews telefonisch und 1 Interview auf Wunsch des Interviewpartners an seinem Arbeitsplatz persönlich geführt ( $\varnothing$ Interviewdauer: 72 Minuten). Die Ergebnisse der Interviews lie- fern einen Überblick über relevante Einrichtungsmerkmale bezogen auf die (1) intendierten Outcomes der Einrichtungen, (2) die Abgrenzung der Einrichtungsformen, (3) den Erstkontakt und die Zuweisung von Patient*innen, (4) die Vergabekriterien für Betreuungsplätze, (5) Versorgungsangebote, (6) die zeitliche Flexibilität der Inanspruchnahme sowie auf (7) Finanzierungsmodalitäten.

\section{Intendierte Outcomes seitens der palliativmedizinischen Tagesklinik/des Tageshospizes}

Das Tageshospiz wird beschrieben als „ein Ort, an den Menschen sehr gerne gehen, [... an] dem sie zur Ruhe kommen, ganz viel schöne Dinge tun können und wissen, sie gehen aber auch wieder nach Hause - also eine Ergänzung zu ihrem Zuhause, zu ihrem normalen Leben, ein neuer Lebensbestandteil“ (E1, Tageshospiz [TH]). Aus Interviews mit Leitungen beider Versorgungsformen erfahren wir, dass die teilstationäre Versorgung ein Verbleiben der Patient*innen im häuslichen Umfeld durch eine Entlastung der Angehörigen ermöglichen soll: „Es ist eine schöne Entlastung für die Angehörigen, die [...] vielleicht auch mal Freiräume brauchen“ (E3, TH). Entsprechend beschreibt die Leitung einer palliativmedizinischen Tagesklinik: „In letzter Konsequenz geht es ja darum, häusliche Versorgungsstrukturen zu stabilisieren“ (E2, Tagesklinik [TK]). Teilstationäre Einrichtungen sollen dabei nicht als Vorbereitung auf eine vollstationäre Aufnahme fungieren; intendiertes Outcome ist, das Sterben im häuslichen Umfeld zu ermöglichen: „Es geht nicht darum, [...] das Tageshospiz vorzuschalten vor ,Ach, dann sehen Sie schon mal wie es dann im Hospiz laufen kann“ und sie dann in das Hospiz zu übernehmen. Das ist nicht mein Ansehen und das sollte nach Möglichkeit auch nicht passieren“ (E4, TH).

\section{Abgrenzung von palliativmedizinischer Tagesklinik und Tageshospiz}

Sowohl zwischen den beiden Formen der teilstationären Betreuung (palliativmedizinische Tagesklinik vs. Tageshospiz) als auch zwischen diesen teilstationären Einrichtungsformen und vollstationären Einrichtungen werden Unterschiede wahrgenommen. Die Leitung einer palliativmedizinischen Tagesklinik beschreibt den eigenen Leistungsschwerpunkt wie folgt: „Unser palliativmedizinischer Therapieschwerpunkt ist ein medizinischer [...] wobei gleichermaßen da diese körperliche Symptomlinderung wie auch die psychosoziale Symptomlinderung wichtig ist“ (E2, TK). Den Unterschied zwischen teil- und vollstationärer Einrichtung erläutert eine Hospizleitung folgendermaßen: „[Das] stationäre [...] Hospiz hat so ein bisschen den Fokus um die Mahlzeiten herum und dann im Tageshospiz geht es auch so ein bisschen um die Tagesgestaltung“ (E4, TH).

\section{Erstkontakt und Zuweisungen}

Die Zuweisung von Tagespatient*innen erfolgt auf verschiedenen Wegen: „Der erste Kontakt erfolgt in der Regel dadurch, dass der zuweisende Arzt anruft“ (E2, TK). Weiter berichtet dieselbe Einrichtungsleitung über folgenden Zugangsweg: „Wir haben [es] aber auch genauso häufig, dass wir Termine [...] vereinbaren, wenn wir palliativmedizinisch konsiliarisch in der Behandlung eben drinnen sind. Das sind aber auch nicht selten $\mathrm{Pa}$ - 
tienten oder Angehörige selbst, die bei uns anrufen“ (E2, TK). Aus einer anderen Tagesklinik wird berichtet: „Die Patienten [werden] angemeldet durch den ambulanten Palliativdienst oder durch die Onkologie oder wir kennen sie von der Palliativstation oder dass sich ein Hausarzt meldet“ (E4, TK). Die Leitung eines Tageshospizes erläutert zu Informationsmaterialien, die von Netzwerkpartnern genutzt werden, um das teilstationäre Angebot vorzustellen: „Wir haben Flyer erstellt, die dann mitgenommen werden können und durch das SAPV-Netzwerk, in dem wir ja auch mit drinnen sind, wird von Pflegekräften dann vermittelt“ (E3, TH).

Vergabe- und Ablehnungskriterien für Betreuungsplätze

Bezogen auf die Vergabekriterien beschreiben Einrichtungsleitungen Parallelen zwischen voll- und teilstationärem Angebot: „Genau die gleiche Voraussetzung, wie für das [voll]stationäre Hospiz: Eine Erkrankung [...], steht ja auch in der Rahmenverordnung [...], die das Leben begrenzt auf Tage, Wochen, vielleicht ein paar Monate“ (E3, TH). Allerdings werden auch - insbesondere auf die Mobilität der Tagesgäste bezogen - Unterschiede beschrieben: „Sie können nur eine tagesklinische Behandlung für jemanden anbieten, der zu Ihnen kommen kann. Also Teilmobilität [...] ist definitiv ein Kriterium und diese Teilmobilität - also zumindest Rollstuhlmobilität - muss gewährleistet sein“ (E2, TK). „Es soll schon so sein, dass die Gäste noch einigermaßen am Gemeinschaftsleben teilnehmen können. [...] Ich sage mal so bis Pflegegrad 3 stellen wir uns Gäste im Tageshospiz vor“ (E3, TH).

\section{Versorgungsangebote der teilstationären Einrichtungen}

Das Leistungsangebot palliativmedizinischer Tageskliniken und Tageshospize umfasst die medizinisch-pflegerische (Untersuchungen, Behandlungen) als auch psychosoziale Versorgung (Therapien, Beratungsgespräche, Freizeitangebote). Diese Angebote werden von eigenem Personal oder ehrenamtlich Mitarbeitenden, Personal angegliederter Einrichtungen sowie externen Kooperationspartnern durchgeführt. Dabei sind die Angebote palliativmedizinischer Tageskliniken eher medizinisch ausgerichtet, während in Tageshospizen psychosoziale Unterstützungsangebote (inkl. Freizeitangeboten) überwiegen. So erklärt die Leitung einer palliativmedizinischen Tagesklinik, dass sie sich „darauf beschränken, die Patienten individuell kurzzeitig zu betreuen, medizinische Maßnahmen umzusetzen, um stationäre Aufnahmen zu vermeiden, um Kontakte und Kooperationen mit Psychologie, mit den anderen Fachabteilungen zu machen, aber auch kleinere Eingriffe, um wie gesagt die stationären Aufnahmen zu vermeiden“ (E5, TK). Durch die Anbindung an ein Krankenhaus können für die Behandlung in einer Tagesklinik neben den Palliativmediziner*innen „konsiliarisch ärztliche Kollegen andere[r] Fachdisziplinen hinzugezogen werden“ (E2, TK).

Die medizinische Versorgung in Tageshospizen erfolgt üblicherweise durch Hausärztinnen und -ärzte sowie durch Palliativmediziner*innen, die regelmäßig visitieren oder bei Bedarf hinzugezogen werden. Beide Einrichtungsarten bieten „Symptomlinderung an im psychosozialen Bereich [...]: Das ist die Psychotherapie, Psychoonkologie, das sind die Kreativtherapeutin- nen mit Musik- und Kunsttherapie“ (E1, TH). Im Tageshospiz liegt ein Schwerpunkt auf der Tagesgestaltung und der Gemeinschaft mit anderen Erkrankten: „Die Möglichkeit [zu] haben, am Tag an einem bestimmten Programm teilzunehmen, therapeutisch oder auch einfach nur mit Pflegekräften gemeinsam den Tag gestalten [zu] können, [...] mit anderen in Kontakt zu treten [ist] ganz wichtig, weil viele [...] aus der Häuslichkeit [kommen], kommen da auch nicht mehr richtig raus“ (E3, TH).

\section{Möglichkeiten zeitlich flexibler Inanspruchnahme}

Einrichtungsleitungen von Tageshospizen erörtern, dass individuelle Absprachen bezogen auf die Inanspruchnahme der teilstationären Versorgung mit den Patient*innen erfolgen. Eine Tageshospizleitung betont, dass das „ein offenes Angebot ist, ob man jetzt von Montag bis Freitag kommt [...] oder man sagt: ,Ich möchte doch nur 1 oder 2 Tage kommen“ “ (E4, TH). Individuelle Vereinbarungen werden auch auf täglicher Basis getroffen. Patient*innen können selbst entscheiden, ob sie an einem Tag kommen möchten „Meistens ist es so, dass die Angehörigen anrufen oder der Gast selber und sagt: ,Mensch, heute geht es mir gar nicht, ich komme nicht. Ich komme gar nicht hoch.' [...] Das hatten wir schon, dass ein Gast sich selber abgemeldet hat oder dass Angehörige gesagt haben: ,Mensch da ist irgendwas nicht so richtig, wir fahren doch nochmal in die Klinik' oder so. Also das war dann eher spontan“ (E3, TH). Auch die tägliche Anwesenheitszeit kann variieren: „Wir gucken wie lange ein Gast auch aushält. Das ist ja manchmal auch sehr anstrengend, wenn man so 3, 4, 5 Stunden in einer Einrichtung ist. Das ist für manchen schon die Höchstgrenze, was er so kann. Dann würden wir den Tag eben auch verkürzen mit Absprache der Angehörigen oder der Pflegedienste zu Hause“ (E3, TH). Anwesenheitsintervalle in palliativmedizinischen Tageskliniken können sich ebenfalls für eine Patientin oder einen Patienten verändern: „Es ist auch so, dass die in der Zeit mal häufiger kommen, mal gar nicht [...] und das geht zum Teil über Jahre“ (E5, TK). Im Unterschied zu manchem Tageshospiz, ist in der palliativmedizinischen Tagesklinik keine tägliche Anwesenheit vorgesehen: „Tagesklinische Behandlung bedeutet ja nicht, dass die Patienten jeden Tag kommen, sondern in entsprechend abgestimmtem Intervall und das hängt schwerpunktmäßig von der Symptomlast ab“ (E2, TK).

\section{Finanzierung}

Leitungen der palliativmedizinischen Tageskliniken als auch der Tageshospize berichten über die Finanzierung ihres teilstationären Angebots durch Tagessätze: „Die Tagesklinik finanziert sich ansonsten durch tagesgleiche Sätze. Also wir kriegen ein tagesbezogenes Entgelt, das mit den Kostenträgern verhandelt wird“ (E6, TK). Ähnlich wird für das Tageshospiz beschrieben: „Der Tagessatz finanziert sich über $95 \%$ Tagessatz Krankenkasse und $5 \%$ müssen wir als Spenden dazu bringen“ (E3, TH). Eine tageweise Abrechnung wird in den Interviews als wünschenswert benannt, da „dann egal ist, ob es jetzt in der einen Woche montags und donnerstags ist und in der anderen Woche mittwochs und freitags“ (E4, TH). Das Einbringen von Eigenmitteln wird als Herausforderung gesehen: „Wir verhandeln [...] gemäß der Rahmenvereinbarung, [...] in der festgelegt wird, ich sage 
- Tab. 1 Anzahl der Tageshospize und palliativmedizinischen Tageskliniken nach ausgewählten Einrichtungsmerkmalen.

\begin{tabular}{|c|c|c|c|c|}
\hline \multicolumn{2}{|l|}{ Variable } & Tageshospize & Tageskliniken & gesamt \\
\hline \multicolumn{5}{|l|}{ Eröffnung } \\
\hline \multirow{6}{*}{ Jahr der Eröffnung bzw. Stand der Planung } & vor 2007 & 2 & - & 2 \\
\hline & 2007-2012 & - & 3 & 3 \\
\hline & $2013-2017$ & 2 & - & 2 \\
\hline & seit 2018 & 6 & - & 6 \\
\hline & im Aufbau & 4 & 1 & 5 \\
\hline & in Planung & 8 & 2 & 10 \\
\hline \multicolumn{5}{|l|}{ Versorgungszeiten } \\
\hline \multirow[t]{6}{*}{ geöffnete Tage } & 1-2 Tage/Monat & 1 & - & 1 \\
\hline & 1-2 Tage/Woche & 6 & 1 & 7 \\
\hline & 3-4 Tage/Woche & 2 & - & 2 \\
\hline & >4 Tage/Woche (täglich) & 10 & 4 & 14 \\
\hline & noch nicht entschieden & 2 & 1 & 3 \\
\hline & keine Angabe & 1 & - & 1 \\
\hline \multirow[t]{4}{*}{ geöffnete Stunden } & $<4$ Stunden & - & 1 & 1 \\
\hline & 4-8 Stunden & 14 & 4 & 18 \\
\hline & > 8 Stunden & 6 & - & 6 \\
\hline & noch nicht entschieden & 2 & 1 & 3 \\
\hline \multicolumn{5}{|l|}{ Finanzierung } \\
\hline \multirow[t]{2}{*}{ Finanzierung durch Krankenkasse } & ja & 16 & 5 & 21 \\
\hline & nein & 6 & 1 & 7 \\
\hline \multirow[t]{8}{*}{ Anteil Spenden an Finanzierung } & $0 \%$ & - & - & - \\
\hline & $>0 \%$ bis $\leq 5 \%$ & 3 & 3 & 6 \\
\hline & $>5 \%$ bis $\leq 25 \%$ & 10 & 1 & 11 \\
\hline & $>25 \%$ bis $\leq 50 \%$ & 3 & - & 3 \\
\hline & $>50 \%$ bis $\leq 75 \%$ & - & - & - \\
\hline & $>75 \%$ bis $<100 \%$ & 2 & - & 2 \\
\hline & $100 \%$ & 1 & 1 & 2 \\
\hline & unbekannt/keine Angabe & 3 & 1 & 4 \\
\hline \multicolumn{5}{|l|}{ Vernetzung } \\
\hline \multirow[t]{3}{*}{ Teil eines hospizlich-palliativen Netzwerks } & ja & 20 & 5 & 25 \\
\hline & nein & 2 & 1 & 3 \\
\hline & $\mathbf{n}$ & 22 & 6 & 28 \\
\hline
\end{tabular}

jetzt mal: Wir brauchen 1 Hospizleitung, 1 Pflegedienstleitung, 10 Krankenschwestern, 2 Köche, 1 Haustechniker und 1 Reinigung. [...] Aufgrund dieser Rahmenvereinbarung verhandeln wir mit den Krankenkassen einen täglichen Bedarfssatz. Von diesem Bedarfssatz bekommen wir aber nur 95\%. [...] Für das, was unsere alltägliche Arbeit ausmacht, müssen wir schon für jeden Tag, für jeden Patienten $5 \%$ an Spenden einwerben. Wir haben aber keinen Menschen für die Öffentlichkeitsarbeit. Der wiederrum wird nicht bezahlt" (E1, TH). Nicht alle Einrichtungen rechnen ihre Leistungen über Krankenkassen ab: „mit Unterstützung des Fördervereins, wie gesagt, bis heute nicht von den Kassen finanziert" (E5, TK). 
\Tab.2 Kapazitäten von Tageshospizen und palliativmedizinischen Tageskliniken.

\begin{tabular}{|c|c|c|c|c|}
\hline \multicolumn{2}{|l|}{ Variable } & Tageshospize & Tageskliniken & gesamt \\
\hline \multirow[t]{3}{*}{ Anzahl der Betreuungsplätze } & $\mathrm{n}$ & 20 & 6 & 26 \\
\hline & $\mathrm{M}( \pm S D)$ & $6,6(2,5)$ & $3,3(1,6)$ & $5,9(2,7)$ \\
\hline & $\mathrm{x}_{\min }-\mathrm{x}_{\max }$ & $2-13$ & $2-6$ & $2-13$ \\
\hline \multirow{3}{*}{$\begin{array}{l}\text { Anzahl tatsächlich } \\
\text { versorgter Patient }{ }^{*} \text { innen } \\
\text { (nur Einrichtungen } \\
\text { in Betrieb) }\end{array}$} & $n$ & 9 & 3 & 12 \\
\hline & $M( \pm S D)$ & $4,1(2,5)$ & $5,0(1,0)$ & $4,3(2,2)$ \\
\hline & $x_{\min }-x_{\max }$ & $2-10$ & $4-6$ & $2-10$ \\
\hline
\end{tabular}

\section{Ergebnisse der deskriptiven Analyse der quantitativen bundesweiten Befragung}

Von den recherchierten 25 Tageshospizen nahmen 22, von den 8 palliativmedizinischen Tageskliniken 6 an der Befragung teil, sodass insgesamt ein Rücklauf von $84,8 \%$ erzielt wurde ( $n=$ 28). Von den befragten Tageshospizen haben 10 bereits ihren Betrieb aufgenommen, 4 befinden sich zum Erhebungszeitpunkt im Aufbau, sodass ein konkreter Eröffnungstermin absehbar ist ( $\downarrow$ Tab. 1 ). Weitere 8 Tageshospize sind nach eigener Angabe noch in Planung, d.h. ein konkreter Aufbau hat zum Zeitpunkt der Befragung noch nicht begonnen. Von den befragten Tageskliniken sind 3 bereits in Betrieb, 1 im Aufbau und 2 noch in Planung. Dabei gibt es sowohl Einrichtungen, die nur an bestimmten Tagen in der Woche oder im Monat geöffnet haben als auch solche mit einem Betreuungsangebot von 5 Tagen pro Woche und mehr. An den geöffneten Tagen kann - mit einer Ausnahme - von einer Betreuungszeit von mindestens 4 Stunden ausgegangen werden.

Obwohl keine teilstationäre Einrichtung ohne Spenden betrieben wird, variiert der Anteil der Spenden an der Gesamtfinanzierung zwischen den Einrichtungen stark: Während 6 Einrichtungen Spenden von höchstens $5 \%$ akquirieren - für von Krankenkassen bezuschusste Hospize ist nach §39a Abs. 1 SGB $\checkmark$ ein Eigenanteil von $5 \%$ des tagesbezogenen Bedarfssatzes vorgesehen [24] -, stellen Spenden bei anderen die wesentliche oder sogar alleinige Finanzierungsquelle dar. Finanzielle Zuschüsse durch Krankenkassen erhalten 75\% der Einrichtungen.

In den 6 befragten Tageskliniken stehen zwischen 2 und 6 Behandlungsplätze zur Verfügung ( $\varnothing 3,3$; > Tab.2). De facto versorgen die 3 bereits in Betrieb genommenen Tageskliniken durchschnittlich fast doppelt so viele Patient*innen $(\varnothing 5,0)$ als offiziell Plätze zur Verfügung stehen (Ø 2,7). Die Anzahl der Betreuungsplätze in 21 befragten Tageshospizen variiert meist zwischen 2 und 8, ein Tageshospiz bietet 20 Plätze an. In den bereits betriebenen Tageshospizen werden durchschnittlich 4,1 Patient*innen an einem geöffneten Tag versorgt.

Die meisten teilstationären Einrichtungen sind Teil eines hospizlich-palliativen Netzwerks ( $n=25$; $>$ Tab. 1 ) und organisatorisch eher selten eigenständig: Alle Tageskliniken und 72,7\% der Tageshospize sind an eine andere Einrichtung angeschlos- sen ( $\triangleright$ Tab. 3). Bei Tageskliniken handelt es sich dabei in der Regel um Kliniken, bei Tageshospizen häufig um vollstationäre Hospize oder ambulante Hospizdienste. Die Angliederung wirkt sich durch die räumliche Nähe auf die gemeinsame Ressourcennutzung hinsichtlich Räumlichkeiten und Personaleinsatz aus. Lediglich 6 Einrichtungen sind baulich von der angeschlossenen Institution getrennt. Zahlreiche Berufsgruppen, die bereits in der angegliederten Einrichtung oder im angegliederten Dienst angestellt sind, werden ebenfalls in der teilstationären Versorgung eingesetzt. Wesentliche Komponente aller teilstationären Einrichtungen ist die Unterstützung durch Ehrenamtliche: In Tageshospizen sind durchschnittlich 9,5 und in Tageskliniken 2,8 ehrenamtlich Mitarbeitende tätig.

Von den Befragten aus 12 bereits bestehenden Einrichtungen gibt die Mehrheit $(n=7)$ an, dass durch die Betreuung im Tageshospiz bzw. die Behandlung in der palliativmedizinischen Tagesklinik vollstationäre Aufnahmen oft vermieden werden können ( $\triangleright$ Tab.4). Der Wunsch, vollstationäre Aufnahmen zu vermeiden, wird von allen Befragten aus palliativmedizinischen Tageskliniken ( $n=3)$ und von den meisten Befragten aus Tageshospizen $(n=6)$ als ziemlich bzw. völlig zutreffender Grund der Patient*innen für den Besuch der Tageseinrichtungen wahrgenommen. Hingegen führen nur Befragte aus Tageshospizen den Besuch ihrer Tagesgäste zurück auf einen Wunsch nach sozialen Kontakten (trifft ziemlich/völlig zu: $n=9$ ) und Freizeitaktivitäten (trifft ziemlich/völlig zu: $n=3$, trifft teils-teils zu: $n=5$ ).

Der erste Kontakt zu einer palliativmedizinischen Tagesklinik oder einem Tageshospiz wird vorrangig über SAPV-Dienste (oft: $n=4$, sehr oft: $n=4$ ), Kliniken und Krankenhäuser (oft: $n=$ 4, sehr oft: $n=3$ ) sowie An- und Zugehörige (oft: $n=4$, sehr oft: $n=2$ ) hergestellt ( $>$ Tab.5). Eine Kontaktaufnahme durch die Patient*innen selbst findet vergleichsweise weniger häufig statt (oft: $n=3$, sehr oft: $n=0$ ).

In der eigenen Häuslichkeit werden Patient*innen vor allem durch An- und Zugehörige versorgt (oft: $n=4$, sehr oft: $n=8$ ). Parallel zur teilstationären Versorgung unterstützen weiterhin Hausärztinnen und -ärzte, SAPV-Dienste sowie ambulante Pflege- und Hospizdienste. Eine teilstationäre Betreuung endet am häufigsten aufgrund des Todes der Patient*innen (oft: $n=6$, sehr oft: $n=1$ ) oder der Verlegung in andere vollstationäre Betreuungsformen (oft: $n=5$, sehr oft: $n=1$ ). 
- Tab.3 Anzahl von Einrichtungen mit Angliederung an andere Versorger und Charakteristika der Angliederung.

\begin{tabular}{|c|c|c|c|c|}
\hline \multicolumn{2}{|l|}{ Variable } & Tageshospize & Tageskliniken & gesamt \\
\hline \multicolumn{5}{|c|}{ Angliederung an andere Einrichtung } \\
\hline \multicolumn{2}{|l|}{ ja } & 16 & 6 & 22 \\
\hline \multicolumn{2}{|l|}{ nein } & 2 & - & 2 \\
\hline \multicolumn{2}{|l|}{ ist noch nicht entschieden } & 4 & - & 4 \\
\hline \multicolumn{2}{|l|}{ n } & 22 & 6 & 28 \\
\hline \multicolumn{5}{|c|}{ Art der angegliederten Einrichtung/des angegliederten Dienstes } \\
\hline \multirow[t]{5}{*}{ Mehrfachnennungen möglich } & stationäres Hospiz & 13 & 1 & 14 \\
\hline & Klinik/Krankenhaus & 1 & 5 & 6 \\
\hline & ambulanter Pflegedienst & 1 & - & 1 \\
\hline & ambulanter Hospizdienst & 8 & - & 8 \\
\hline & sonstige Angliederung & 6 & 1 & 7 \\
\hline \multicolumn{5}{|c|}{ gemeinsame Nutzung mit angegliederter Einrichtung/angegliedertem Dienst } \\
\hline \multirow[t]{4}{*}{ Mehrfachnennungen möglich } & aller Räumlichkeiten & 3 & 4 & 7 \\
\hline & Dienstzimmer/Büros & 4 & 3 & 7 \\
\hline & $\begin{array}{l}\text { eigener Bereich innerhalb } \\
\text { angegliederter Einrichtung }\end{array}$ & 11 & 4 & 15 \\
\hline & baulich getrennt & 6 & - & 6 \\
\hline \multicolumn{5}{|c|}{$\begin{array}{l}\text { in beiden Bereichen eingesetzte Berufsgruppen } \\
\text { (Tagesangebot und angegliederte Einrichtung/angegliederter Dienst) }\end{array}$} \\
\hline \multirow[t]{11}{*}{ Mehrfachnennungen möglich } & Leitung/Koordination & 12 & 6 & 18 \\
\hline & $\begin{array}{l}\text { Pflegekraft mit Palliative-Care- } \\
\text { Weiterbildung }\end{array}$ & 8 & 4 & 12 \\
\hline & $\begin{array}{l}\text { Pflegekraft ohne Palliative-Care- } \\
\text { Weiterbildung }\end{array}$ & 4 & 2 & 6 \\
\hline & Arzt/Ärztin & 3 & 5 & 8 \\
\hline & Psychologe/Psychologin & 1 & 5 & 6 \\
\hline & Sozialarbeiter ${ }^{*}$ in & 11 & 6 & 17 \\
\hline & Seelsorger*in & 5 & 6 & 11 \\
\hline & Kreativtherapeut*in & 4 & 3 & 7 \\
\hline & Physio-/Ergotherapeut* in & 1 & 6 & 7 \\
\hline & Hauswirtschaft/Küche & 11 & 5 & 16 \\
\hline & keine Angabe & 1 & - & 1 \\
\hline \multicolumn{2}{|c|}{ n (nur angegliederte Einrichtungen) } & 16 & 6 & 22 \\
\hline
\end{tabular}

\section{Diskussion}

Diese Arbeit berichtet erstmalig für Deutschland den Bestand palliativmedizinischer Tageskliniken und Tageshospize und beschreibt deren Charakteristika. Die Kombination qualitativer und quantitativer Daten ermöglicht, ein umfassendes Bild über den Bestand palliativmedizinischer Tageskliniken und Tageshospize zu erhalten. Zudem lässt der hohe Rücklauf (84,8\%) einen umfangreichen Überblick einrichtungsbezogener Merkmale der bestehenden und in Planung befindlichen Tageshospize und palliativmedizinischen Tageskliniken in Deutschland zu. Daten, die mit in Planung befindlichen Einrichtungen erhoben wurden, sind jedoch unter Vorbehalt zu betrachten, da Aspekte in ihrer praktischen Umsetzung von der Planung abweichen können. 
- Tab.4 Gründe der Inanspruchnahme und Beendigung teilstationärer Leistungen, Häufigkeitsangaben und Zustimmungen von Einrichtungsleitungen auf 5-stufiger Antwortskala, nur Einrichtungen in Betrieb $(n=12)^{\text {a }}$.

\begin{tabular}{|c|c|c|c|c|c|c|c|}
\hline & & $\begin{array}{l}\text { sehr } \\
\text { selten }\end{array}$ & selten & $\begin{array}{l}\text { gelegent- } \\
\text { lich }\end{array}$ & oft & sehr oft & $\begin{array}{l}\text { keine } \\
\text { Angabe }\end{array}$ \\
\hline \multicolumn{2}{|l|}{$\begin{array}{l}\text { Häufigkeit der Vermeidung vollstationärer } \\
\text { Aufnahmen }\end{array}$} & - & - & 2 & 7 & 1 & 2 \\
\hline \multicolumn{8}{|l|}{ Häufigkeit der Beendigungsgründe } \\
\hline \multicolumn{2}{|l|}{ Tod } & 1 & - & 2 & 6 & 1 & 2 \\
\hline \multicolumn{2}{|c|}{ Verlegung in eine vollstationäre Betreuungsform } & - & 3 & 3 & 5 & 1 & - \\
\hline \multicolumn{2}{|c|}{ Beschränkung auf häusliche ambulante Pflege } & 3 & - & 1 & 2 & - & 6 \\
\hline \multicolumn{8}{|c|}{ Gründe für Inanspruchnahme der teilstationären Versorgung } \\
\hline & \multicolumn{3}{|c|}{ palliativmedizinische Tageskliniken } & \multicolumn{4}{|c|}{ Tageshospize } \\
\hline & $\begin{array}{l}\text { trifft gar } \\
\text { nicht/ } \\
\text { wenig zu }\end{array}$ & $\begin{array}{l}\text { trifft teils- } \\
\text { teils zu }\end{array}$ & $\begin{array}{l}\text { trifft } \\
\text { ziemlich/ } \\
\text { völlig zu }\end{array}$ & $\begin{array}{l}\text { trifft gar } \\
\text { nicht/ } \\
\text { wenig zu }\end{array}$ & $\begin{array}{l}\text { trifft teils- } \\
\text { teils zu }\end{array}$ & $\begin{array}{l}\text { trifft } \\
\text { ziemlich/ } \\
\text { völlig zu }\end{array}$ & $\begin{array}{l}\text { keine } \\
\text { Angabe }\end{array}$ \\
\hline Vermeidung vollstationärer Aufnahme & - & - & 3 & 1 & 1 & 6 & 1 \\
\hline Entlastung der An- und Zugehörigen & 1 & 1 & 1 & - & 2 & 7 & - \\
\hline Wunsch nach sozialen Kontakten & 2 & 1 & - & - & - & 9 & - \\
\hline $\begin{array}{l}\text { Wunsch nach psychosozialer Beratung/ } \\
\text { Unterstützung }\end{array}$ & 1 & 1 & 1 & 2 & 2 & 5 & - \\
\hline Wunsch nach medizinischer Versorgung & - & - & 3 & 3 & 4 & 2 & - \\
\hline Wunsch nach pflegerischer Versorgung & - & 3 & - & 4 & 2 & 3 & - \\
\hline Wunsch nach Freizeitaktivitäten & 3 & - & - & 1 & 5 & 3 & - \\
\hline$n$ & \multicolumn{3}{|l|}{3} & \multicolumn{4}{|l|}{9} \\
\hline
\end{tabular}

Obwohl es in Deutschland im Vergleich zu anderen Ländern, wie etwa Großbritannien, noch relativ wenige teilstationäre hospizlich-palliative Versorgungseinrichtungen gibt, wird anhand der hohen Anzahl von Einrichtungen, die derzeit geplant werden, sich bereits im Aufbau befinden oder erst kürzlich eröffnet wurden, die Dynamik in diesem Versorgungsbereich deutlich. Die Angebote palliativmedizinischer Tageskliniken und Tageshospize unterscheiden sich dahingehend voneinander, dass palliativmedizinische Tageskliniken einen besonderen Schwerpunkt auf die medizinische Versorgung legen, während Tageshospize stärker psychosoziale Aspekte sowie Freizeitangebote fokussieren. Entsprechend sind aus Großbritannien 2 Modelle der hospizlich-palliativen Tagesversorgung (ein medizinisches, ein soziales) bekannt, wobei immer mehr Einrichtungen als Mischung aus beiden Modellen beschrieben werden können [25-27].

Die Ergebnisse der vorliegenden Studie konnten erste Hinweise dafür liefern, dass die in Deutschland vorhandenen Angebote angenommen werden: So versorgen die Tageskliniken in der Befragung mehr Patient*innen als es die Kapazitäten eigentlich zulassen. Tatsächlich bleibt die Anzahl durchschnittlich versorgter Tagesgäste in den Tageshospizen hinter der Anzahl vorhandener Versorgungsplätze zurück. Das gleiche Phänomen zeigte sich bereits in einer früheren Studie für Großbri- tannien, obwohl Einrichtungen dort zum Teil sogar Wartelisten führten [28]. Dies hängt möglicherweise mit der Organisation der Platz- und Terminvergabe zusammen: Tageskliniken bedienen einen großen Pool an Patient*innen, die sie zu regelmäßigen Terminen oder - bei hohem Leidensdruck - auch kurzfristig behandeln. Tageshospize hingegen vergeben in der Regel pro Patient*in einen festen Platz in ihrer Einrichtung, der flexibel und in Abhängigkeit des Wohlbefindens und anderer Termine von den Patient*innen genutzt werden kann. Seltener operieren Tageshospize nach dem Konzept der „offenen Tür“, wobei sie ihr Angebot an bestimmten Tagen in der Woche oder im Monat anbieten und den Betroffenen eine spontane Teilnahme ermöglichen.

Wie umfangreich und regelmäßig ein Angebot stattfinden kann, hängt nicht zuletzt stark von den finanziellen Voraussetzungen ab. Grundsätzlich können 2 Finanzierungsmodelle unterschieden werden: In dem einen wird eine teilstationäre Fallpauschale über die Krankenkassen erhalten und damit ein erheblicher Teil der teilstationären Arbeit finanziert. In dem anderen verfügt die Einrichtung über keinen Versorgungsvertrag und ist maßgeblich spendenfinanziert. Dabei zeigt sich bei den Tageshospizen - durch die obligatorische Eigenleistung von $5 \%$ [24] - wie auch bei den Tageskliniken, bei denen die durchschnittliche Belegung die Anzahl gegenfinanzierter Plätze fast 
Tab. 5 Interaktion von Tageshospizen und palliativmedizinischen Tageskliniken mit anderen Versorgern, Häufigkeitsangaben von Einrichtungsleitungen auf 5-stufiger Antwortskala, nur Einrichtungen in Betrieb $(n=12)^{\text {a. }}$.

\begin{tabular}{|c|c|c|c|c|c|c|}
\hline & sehr selten & selten & gelegentlich & oft & sehr oft & keine Angabe \\
\hline \multicolumn{7}{|c|}{ Häufigkeit des ersten Kontakts durch } \\
\hline SAPV-Dienste & - & 1 & 2 & 4 & 4 & 1 \\
\hline An- und Zugehörige & - & 1 & 5 & 4 & 2 & - \\
\hline ambulante Hospizdienste & - & 2 & 6 & 1 & 3 & - \\
\hline Klinik/Krankenhaus & 2 & 2 & 1 & 4 & 3 & - \\
\hline Hausärzte/-ärztinnen & - & 2 & 6 & 3 & 1 & - \\
\hline Patienten selbst & 2 & - & 7 & 3 & - & - \\
\hline ambulanter Pflegedienst & 1 & 5 & 3 & 2 & 1 & - \\
\hline \multicolumn{7}{|c|}{ Häufigkeit der parallelen Versorgung } \\
\hline durch An- und Zugehörige & - & - & - & 4 & 8 & - \\
\hline durch Hausarzt/-ärztin & - & - & 1 & 7 & 4 & - \\
\hline ambulant durch Pflegedienst & - & - & 4 & 4 & 4 & - \\
\hline ambulant durch Hospizdienst & - & 1 & 3 & 6 & 2 & - \\
\hline durch SAPV-Dienst & 2 & - & - & 8 & 1 & 1 \\
\hline
\end{tabular}

um das Doppelte übersteigt, dass alle Einrichtungen auf zusätzliche Finanzierungsquellen angewiesen sind.

Die teilstationäre Versorgung losgelöst von anderen ambulanten und stationären Versorgungsangeboten zu betrachten, würde der Wirklichkeit nicht gerecht werden, da verschiedene Versorgungsformen ineinander greifen [29]. So zeigen auch unsere Daten, dass Patient*innen häufig von unterschiedlichen Versorger*innen parallel betreut werden. Sowohl die Interviews mit Leitungspersonen als auch die Auswertung der quantitativen Befragung zeigen, dass oftmals bereits versorgende Akteurinnen und Akteure den ersten Kontakt zum Tagesangebot vermitteln, da die Betroffenen von den Versorgungsmöglichkeiten zunächst einmal Kenntnis erlangen müssen. Um eine ganzheitliche, sich optimal ergänzende Versorgungssituation für die Betroffenen zu erreichen, scheint eine gute Vernetzung aller an der Versorgung beteiligten Akteurinnen und Akteure unerlässlich. Eine große Bedeutung kommt somit hospizlich-palliativen Netzwerken [29, 30] zu, in denen 25 der befragten Einrichtungen mit anderen regionalen Versorgern vernetzt sind. Tageshospize und palliativmedizinische Tageskliniken, die an eine bereits bestehende Einrichtung angegliedert sind, profitieren von bereits etablierten Netzwerkstrukturen. Zudem ergeben sich durch eine Angliederung weitere Synergieeffekte, wie die gemeinsame Nutzung von Räumlichkeiten sowie der Austausch von Personal [31].

\section{Fazit}

Die teilstationäre hospizlich-palliative Versorgung in Deutschland ist ein diverses und dynamisches Feld mit vielfältigen Ein- richtungsmodellen und aktuell neu entstehenden Einrichtungen. Tageshospize und palliativmedizinische Tageskliniken können eine Versorgungslücke schwerstkranker Menschen am Lebensende schließen, indem tagsüber eine umfängliche medizinisch-pflegerische und psychosoziale Versorgung angeboten wird. Tageshospize wirken zudem gezielt sozialer Isolation entgegen. Palliativmedizinische Tageskliniken ermöglichen eine multiprofessionelle Behandlung im Krankenhaus ohne eine stationäre Aufnahme erforderlich zu machen und können somit ein wichtiger Baustein der palliativmedizinischen Frühintegration sein. Tagesangebote können Patient* innen durch eine Entlastung ihrer Angehörigen ermöglichen, weiterhin im eigenen Zuhause zu wohnen und ihre Selbstständigkeit zu bewahren.

In zukünftigen Forschungsprojekten sollte die Perspektive der Patient*innen mehr in den Blick genommen werden, um Angebote noch bedürfnisorientierter in die vorhandene Versorgungslandschaft integrieren zu können.

\section{Einhaltung ethischer Richtlinien}

Die hier präsentierte Erhebung erhielt im Rahmen der Studie „ABPATITE“ am 10.02.2020 ein positives Votum der Ethikkommission der Medizinischen Hochschule Hannover (8892_BO_ S_2020).

\section{Danksagung}

Das Projektteam dankt den Leitungspersonen palliativmedizinischer Tageskliniken und Tageshospize für ihre Teilnahme an den Erhebungen. 
Interessenkonflikt

Die Autorinnen/Autoren geben an, dass kein Interessenkonflikt besteht.

\section{Förderung}

Das Projekt ABPATITE wird vom Innovationsfonds beim Gemeinsamen Bundesausschuss finanziert (Förderkennzeichen: 01VSF19034, Laufzeit: 04/2020-09/2022).

\section{Literatur}

[1] Eve A, Smith AM, Tebbit P. Hospice and palliative care in the UK 19945 , including a summary of trends 1990-5. Palliat Med 1997; 11: $31-$ 43

[2] Goodwin DM, Higginson IJ, Myers K et al. Effectiveness of palliative day care in improving pain, symptom control, and quality of life. J Pain Symptom Manag 2003; 25: 202-212

[3] Davies E, Higginson IJ. Systematic review of specialist palliative daycare for adults with cancer. Support Care Cancer 2005; 13: 607-627

[4] Eve A, Smith AM. Palliative care services in Britain and Ireland - update 1991. Palliat Med 1994; 8: 19-27

[5] Corr CA, Corr DM. Adult hospice day care. Death Stud 1992; 16: 155171

[6] Gagnon B. Palliative day-care centers and day hospitals. In: Bruera E, Higginson I, von Gunten CF, eds. Textbook of palliative medicine and supportive care. 2. Aufl. Boca Raton, FL: CRC Press; 2015: 293-303

[7] Kilonzo I, Lucey M, Twomey F. Implementing outcome measures within an enhanced palliative care day care model. J Pain Symptom Manag 2015; 50: 419-423

[8] Eisl CS, Borovnyak E. Tageshospiz - „(Missing) Link“ in der Hospiz- und Palliativversorgung? Ein neuer österreichischer Weg In: Eichner E, Hornke I, Sitte T, eds. Ambulante Palliativversorgung. Ein Ratgeber. Fulda: Deutscher PalliativVerlag; 2014: 66-71

[9] Bouleuc C. Supportive care day hospital: A new tool for palliative care in cancer centers. J Clin Oncol 2017; 35 (Suppl. 31): 170

[10] Distelmans W, Bauwens S, Storme G et al. Palliative day care in Belgium: first observations. Eur J Palliat Care 2005; 12: 170-173

[11] Deutsche Krebsgesellschaft, Deutsche Krebshilfe, AWMF. Leitlinienprogramm Onkologie. Erweiterte S3-Leitlinie Palliativmedizin für $\mathrm{Pa}$ tienten mit einer nicht-heilbaren Krebserkrankung. Langversion 2.2. AWMF-Registernummer: 128/001OL. 2020: https://www.leitlinienprogramm-onkologie.de/leitlinien/palliativmedizin/; Zugegriffen: 14.12.2020

[12] Simon ST, Pralong A, Welling U et al. Versorgungsstrukturen in der Palliativmedizin. Behandlungspfad für Patienten mit einer nichtheilbaren Krebserkrankung. Internist 2016; 57: 953-958

[13] Deutscher Hospiz- und PalliativVerband e. V. Zahlen und Fakten. 2020: https://www.dhpv.de/zahlen_daten_fakten.html; Zugegriffen: 14.12.2020
[14] Institut für Allgemeinmedizin, Medizinische Hochschule Hannover. ABPATITE - Verbesserung der Versorgung von Patienten mit unheilbaren Erkrankungen: Analyse des Bestands und des Bedarfs für palliativmedizinische Tageskliniken und Tageshospize sowie Empfehlungen zur Versorgungsplanung. 2020: https://www.mhh.de/allgmed/ abpatite; Zugegriffen: 14.12.2020

[15] Niederberger M, Peter L. Mixed-Methods-Studien in den Gesundheitswissenschaften. Ein kritischer Überblick. Z Evid Fortb Qual Gesundh Wesen 2018; 133: 9-23

[16] Creswell J, Plano Clark V. Designing and conducting mixed methods research. 2. Aufl. Los Angeles: SAGE; 2011

[17] Heckel M, Kremeike K, Stiel S et al. Gemischt-methodische Studiendesigns in der Palliativforschung. Z Palliativmed 2020; 21: 169-172

[18] Deutsche Gesellschaft für Palliativmedizin e. V. Wegweiser Hospizund Palliativversorgung Deutschland. 2020: https://www.wegweiserhospiz-palliativmedizin.de/de; Zugegriffen: 14.12.2020

[19] Palliativ-Portal. 2020: https://www.palliativ-portal.de/; Zugegriffen: 14.12.2020

[20] Bogner A, Littig B, Menz W. Interviews mit Experten: Eine praxisorientierte Einführung. Wiesbaden: Springer VS; 2014

[21] Guest G, Bunce A, Johnson L. How many interviews are enough? An experiment with data saturation and variability. Field Methods 2006; 18: $59-82$

[22] Mayring P. Qualitative Inhaltsanalyse - Abgrenzungen, Spielarten, Weiterentwicklungen. Forum Qualitative Sozialforschung/Qualitative Social Research 2019. doi:10.17169/fqs-20.3.3343

[23] Mayring P. Qualitative Inhaltsanalyse. In: Flick U, von Kardoff E, Keupp $\mathrm{H}$, von Rosenstiel L et al., eds. Handbuch Qualitative Sozialforschung. Grundlagen, Konzepte, Methoden und Anwendungen. Weinheim: Psychologie Verlags Union; 1991: 209-213

[24] GKV Spitzenverband. Stationäre Hospizversorgung. 2019: https:// www.gkv-spitzenverband.de/krankenversicherung/hospiz_und_palliativversorgung/stat_hospizleistung/stat_hospiz/stat_hospizversorgung.jsp; Zugegriffen: 14. Dezember 2020

[25] de Vries K, Wells J, Plaskota M. Evaluation of an admission and discharge programme at a UK specialist palliative day hospice. Int J Palliat Nurs 2012; 18: 275-281

[26] Dosser I, Nicol JS. What does palliative day care mean to you? Eur J Palliat Care 2006; 13: 152-155

[27] Payne M. Social objectives in cancer care: the example of palliative day care. Eur J Cancer Care 2006; 15: 440-447

[28] Higginson IJ, Hearn J, Myers K et al. Palliative day care: what do services do? Palliat Med 2000; 14: 277-286

[29] Herbst FA, Heckel M, Stiel S et al. Kompetent vernetzt - optimal versorgt! Förderliche Faktoren der Zusammenarbeit in hospizlich-palliativen Versorgungsnetzwerken in Bayern. Bundesgesundheitsbl 2017; 60: $37-44$

[30] Herbst FA, Stiel S, Heckel M et al. Beschreibung und Analyse der Partner in Netzwerken der Hospiz- und Palliativversorgung in Bayern an der Schnittstelle ambulant-stationär: Eine Mixed-Methods-Studie. Z Palliativmed 2017; 18: 310-318

[31] Douglas H-R, Normand CE, Higginson IJ et al. Palliative day care: What does it cost to run a centre and does attendance affect use of other services? Palliat Med 2003; 17: 628-637 